\title{
A hybrid technique combining intramedullary pinning with extramedullary plate fixation in unstable and comminuted radial head fractures following on-table reconstruction
}

\section{Xu Gao}

Qingdao University

Shi-you Dai

Qingdao Municpal Hospital

Hai-Lei Yin ( $\nabla$ stonethrough@yeah.net )

N0.971 Hospital of People's Liberation Army

Fei Li

N0.971 Hospital of People's Liberation Army

Yong-Qiang Sui

Luoyang Ship Material Research Institute

Rui Huang

N0.971 Hospital of People's Liberation Army

Hai-yu Fan

N0.971 Hospital of People's Liberation Army

Research article

Keywords: Radial head, Comminuted fracture, Intramedullary pinnin, On-table reconstruction technique

Posted Date: March 18th, 2021

DOI: https://doi.org/10.21203/rs.3.rs-112213/v2

License: (c) (i) This work is licensed under a Creative Commons Attribution 4.0 International License. Read Full License

Version of Record: A version of this preprint was published at BMC Musculoskeletal Disorders on July 9th, 2021. See the published version at https://doi.org/10.1186/s12891-021-04498-w. 


\section{Abstract}

Background: Management of comminuted radial fractures remains controversial. Currently, the emergence of on-table reconstruction technique has made fixation in comminuted radial head fractures more viable. However, the present study reported an intro-operative unstable displacement from the reconstructed radial head to the neck during plate fixation, characterized by a poor radiocapitellar contact and incongruity between the radial head and neck. A hybrid technique combining with intramedullary pining was performed in our study to restore the normal alignment and maintain the stability of fixation. Therefore, the purpose of this article aimed to prove the feasibility of unstable comminuted radial head fractures treated with the extramedullary plate and intramedullary pinning fixation using titanium elastic nails.

Methods: The clinical, functional and radiographic outcomes of the groups were compared during followup. The radiographic examination was conducted to evaluate the status of bone union, heterotopic ossification and post-traumatic arthritis. The functional assessment was performed to evaluate clinical effects, which included measurements of range of motion (ROM) in the elbow, Visual Analog Scale (VAS) score, Elbow Self-Assessment score (ESAS), Mayo Elbow Performance score (MEPS), and Disabilities of the Arm, Shoulder, and Hand (DASH)Outcome Measure score.

Results: Fifteen patients with unstable fractures were participated with an average follow-up of (37.2 \pm 5.8$)$ months for the experimental group and (33.2 \pm 7.1$)$ months for the control group, respectively. The ROM of the elbow and functional outcomes in the experimental group, including MEPS and DASH, were significantly superior to the control group. However, no significant difference was observed in the mean VAS score between two groups. In the last follow-up, two patients with post-traumatic arthritis rated as grades 1 and two with heterotopic ossifications were observed in the experimental group. In the control group, degenerative changes were observed in four cases (grade 1 in three cases and grade 2 in one case) and heterotopic ossifications rated as grade I were found in two patients.

Conclusion: Collectively, intramedullary pinning with extramedullary plate fixation is feasible in unstable comminuted radial head fractures, which can be considered as a remedial surgery for on-table reconstruction technique.

Trial registration: This study was retrospectively registered

\section{Background}

Mason type III fractures refer to comminuted fractures involving the entire radial head displaced from the shaft [1]. The common surgical treatments for such complicated injuries, such as radial head resection (RHR), radial head arthroplasty (RHA) and open reduction and internal fixation (ORIF), have their merits and demerits, respectively. Since there is lack of long-term and large-volume studies in comminuted radial head fractures, the optimal treatment has not reached a consensus [2-3]. Currently, it has been well recognized that the radial head is an important stabilizer of the forearm and elbow [4]. The emergence of 
specific radial head implants and innovative techniques have made ORIF preferable to preserve the integrity of the fractured radial head [5-6].

On-table reconstruction technique is first described by Businger et al, which provides an optional treatment plan and solves a critical challenge that ORIF is almost impossible to repair comminuted fractures with more fragments [7]. Subsequent published series have proved the availability of this technique. Kiran et al [8] have shown that following Ex situ reconstruction technique, six patients with Mason type-III radial head fractures have a satisfactory motion of elbow with the mean Broberg and Morrey score of 90 points. But non-union occurs in three patients and avascular necrosis in one patient in their study. Latterly, in another study by Everding et al [9], the mean Mayo Elbow Performance score is 82 points and DASH score is 20 points with asymptomatic non-union occurred in one patient, and no signs of avascular necrosis are observed. Additionally, several on-table techniques using polylactide pins or surgical glue are reported with satisfactory to good surgical outcomes [10-11]. Accordingly, this technique provides an optional solution for complicated radial head fractures. Nevertheless, since only a few studies have investigated small amounts of patients with conflicting complication rates, the treatment effects of on-table technique may be discrepant in different cases and its reliability needs to be evaluated cautiously in the practical surgical process.

In the present study, we found that some reconstructed radial heads after mini T-type plate fixation were prone to displace from the radial neck during surgery, characterized by a poor radiocapitellar contact and incongruity between the radial head and neck, and we named it unstable comminuted radial head fractures. In these cases, RHR may be considered as a remedial solution since internal implant has largely lost its effects. However, in the present study, a hybrid technique that combined intramedullary pinning with extramedullary plate fixation was manipulated to maintain the stability of fixation and restore the normal alignment in these unstable fractures, with RHR avoided. Additionally, we also retrospectively compared a series of patients surgically treated with RHR or our hybrid technique due to unstable comminuted radial head fractures, and to further assess the clinical and radiological outcomes of this technique. Therefore, the purpose of this article mainly was to ascertain whether the intramedullary pining fixation could be a remedial operation for unstable comminuted radial head fractures after on-table reconstruction technique.

\section{Methods}

\section{Patients}

Between January 2012 and February 2019, a total of 62 consecutive patients with comminuted radial head fractures were reviewed, retrospectively. Further inclusion criteria were set as follows: (i) Fractures involved entire radial head with three or more fragments, and the radial head was completely displaced from the shaft of radius (Mason type-III); (ii) unstable comminuted radial head fractures were confirmed after on-table reconstruction technique; (iii) definitive treatment consisted of RHR or ORIF by extramedullary plate and intramedullary pinning fixation using titanium elastic nails(TEN); and (iv) the 
follow-up time should be no less than 12 months. The exclusion criteria were previous mobility dysfunction of the injured limb and osseous maturity with closed epiphysis.

Based on the above-mentioned inclusion and exclusion criteria, a total of 15 unstable type-III radial head fractures comprised of 9 males and 6 females were identified at our institution. The demographic data was shown in Table 1. Five cases were caused by falling during sports activities, and 10 cases of injuries were attributed to traffic accidents. According to different surgical solutions, patients were divided into two groups. Seven patients treated with ORIF by extramedullary plate and intramedullary pinning fixation were defined as the experimental group, with eight patients who underwent RHR as the control group. The experimental group consisted of 4 males and 3 females with a mean age of $(35.2 \pm 3.6)$ years, and the control group included 6 males and 2 females with a mean age of (41.6 \pm 2.1$)$ years.

The diagnosis was confirmed by the computed tomography (CT), anteroposterior and lateral fluoroscopic views of the elbow and wrist, so as to identify other concomitant injuries. Two patients in the experimental group had a complex injury patterns: in one patient, there was a posterolateral rotational instability (PLRI) injury of the elbow joint [12], while the other patient had a Monteggia lesion (Jupiter type 2a) [13].

\section{Surgical technique}

All patients were placed in the supine position under general anesthesia. Subsequently, a lateral Kocher approach was selected to access the radial head. The radial head fragments were retrieved from the joint and precisely reconstructed with k-wires and screws on the operation table, followed by placing the reconstructed redial head into the elbow joint (Fig 1). Based on comparing the fracture line at the radial head base with the corresponding line at the radial neck, the radial head was reduced and fixed with 1.5 mm locking mini T-type plates (DOUBLE, mini metal locking plate system, DOUBLE MEDICAL, China), which was placed on the correct lateral position to avoid interfering elbow rotation. With the radial head fixed, stability of the fixation was checked clinically. Strikingly, displacement and tilt could be observed from the reconstructed radial head to the neck, leading to a poor radiocapitellar relationship and loss of normal alignment (Fig 2). Insufficient stability in the fracture site was verified by probing the radial head with fingers or vessel forceps.

In the experimental group, patients were treated with intramedullary pining fixation using $2.0 \mathrm{~mm}$ TEN (Johnson, titanium elastic nails system, Johnson, America). The proximal position of TEN was bent approximately 25-30 degrees. It then was inserted into the medullary space from the proximal radial styloid process. The TEN was hammered slowly upward until the tip was fixed to the subchondral bone, by which the displaced and tilted radial head was repositioned. After achieving satisfactory alignment restoration between the radial head and neck under fluoroscopic control (Fig 3), the end of TEN was cut. Conversely, in the control group, the fixed plate was taken down and the radial head was resected with a bone saw close to the surgical neck. Next, the capsule and annular ligament were sutured with absorbable sutures in both groups, and anchor system was used to repair the avulsion of radial collateral 
ligament. Monteggia fracture was internally fixed with plates and screws through a separate ulnar approach.

\section{Postoperative rehabilitation}

A plaster immobilization was used postoperatively for four weeks in one patient with PLRI in the experimental group, and the remaining patients in both two groups were slung for two weeks, followed by early mobilization. Early active-assisted flexion-extension and pronation-supination were commenced from the first postoperative day. The time to allow weight-bearing or other heavy loading activities were based on the clinical and radiological follow-up assessment.

\section{Evaluation}

The follow-up evaluation included functional and radiographic assessment, which was carried out by two orthopedic surgeons who were not involved in the operation. The functional assessment consisted of measurements of range of motion (ROM) in the elbow, Visual Analog Scale score (VAS), Elbow SelfAssessment score (ESAS) [14], Mayo Elbow Performance score (MEPS) [15] and Disabilities of the Arm, Shoulder, and Hand (DASH)Outcome Measure score [16]. The ESAS mainly evaluated the patient's satisfaction with the elbow using a scale of $1-6$ : 1 , very good; 2 , good; 3 , satisfied; 4 , sufficient; 5 , insufficient; and 6, poor.

Postoperative radiographic assessment was based on both the anteroposterior and lateral fluoroscopic views of the elbow to evaluate the status of bone union, heterotopic ossification, joint incongruity, avascular necrosis, and post-traumatic arthritis until the last follow-up. The degree of heterotopic ossification was assessed according to Hastings and Graham system [17]. Radiographic signs of posttraumatic arthritis were stratified by the Broberg and Morrey system [18]:

- a normal joint as grade 0

- slight narrowing joint space with the occurrence of minimum osteophytes as grade 1

- moderate narrowing joint space and osteophyte formation as grade 2

- disappeared joint space as grade 3

\section{Statistical analysis}

The independent samples t-test was conducted using SPSS 20.0 (SPSS Inc, Chicago, IL, USA) to assess the statistical significance of differences between the two groups. A p-value of less than 0.05 was considered significant.

\section{Results}


Patients participated in the current analysis with an average follow-up of $(37.2 \pm 5.8)$ months for the experimental group and $(33.2 \pm 7.1)$ months for the control group, respectively. Fig. 4-5 illustrate the typical case.

\section{Intraoperative comparison}

The mean operating time for the experimental group was $(128.7 \pm 10.3)$ min and $(122.5 \pm 15.7)$ min for the control group, which was not significantly different $(p=0.523)$. There was also not significant difference in the mean blood loss during surgery, which was $(135.3 \pm 13.2) \mathrm{ml}$ to $(129.1 \pm 16.5) \mathrm{ml}$ for the experimental and control groups, respectively $(\mathrm{p}=0.893$ ). The fluoroscopy times during surgery differed significantly and was $(11.5 \pm 4.2)$ to $(3.7 \pm 1.6)$ between the experimental group and control group $(p=0.000)$. (Table 2$)$

\section{Functional assessment}

The clinical, functional and radiographic outcomes of the groups were summarized in Table 3. The mean

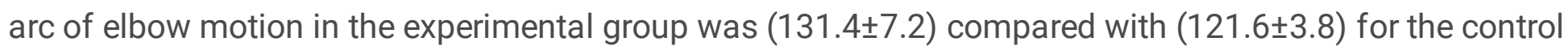
group $(p=0.008)$, and the mean extension deficit was $(13.2 \pm 2.6)$ to $(20.8 \pm 1.9)$ (experiment to control) $(p=0.001)$. There were statistically different between the two groups. However, the average pronation is (73.8 \pm 7.1$)$ to $(74.4 \pm 7.6)(p=0.457)$, and the supination is $(67.7 \pm 4.6)$ to $(70.0 \pm 3.8)(p=0.229)$, which were not statistically different.

One patient in the experimental group following initial treatment of a comminuted radial fracture with PLRI had a flexion deficit of $38^{\circ}$ with the arc of elbow movement of $95^{\circ}$. The ROM of the elbow was insufficient and was considered inferior to the remaining patients. After accepting secondary arthrolysis and systematic rehabilitation, the eventual arc of elbow movement was increased to $134^{\circ}$ with a flexion deficit of $15^{\circ}$. All patients in both two groups could participate in general sports activities without functional limitation of the elbow and joint stiffness.

According to the ESAS, 1 case of very good, 2 cases of good, 3 cases of satisfied, and 1 case of sufficient were rated in the experimental group. In the control group, 3 patients were rated good, 2 satisfied, and 3 sufficient.

The mean VAS score for pain was $(2.5 \pm 0.8)$ for the experimental group and $(3.1 \pm 1.4)$ for the control group ( $p=0.872$ ), respectively, which was not significantly different. Three patients in the experimental group and five in the control group complained of mild pain in the elbow when the weather changes or the injured arm exercises strenuously.However, none of patients considered that the pain affected the daily activities.

Based on the criteria of the MEPS, the mean score of MEPS was (85.6 \pm 7.3$)$ to $(78.2 \pm 2.9)$ between the experimental group and control group at the last follow-up $(p=0.002)$. The mean DASH score was $(9.7 \pm 1.1)$ for the experimental group and $(14.6 \pm 2.8)$ for the control group $(p=0.009)$, respectively. Functional changes of the elbow in two groups were significantly different. 


\section{Radiographic outcomes}

In the experimental group, all reconstructed radial heads survived without signs of avascular necrosis at the final follow-up. Five patients obtained bone union at three months after surgery, with a normal alignment between the axis of radial head and shaft of radius. Two patients appeared as a delayed union and eventually healed six to eight months postoperatively. Loss of reduction or failure in fixation was not observed. Mild signs of post-traumatic arthritis (grades 1) were observed in two patients, and heterotopic ossifications rated as grade I were found in three patients. In the control group, degenerative changes in elbow rated as grade 1 were observed in three patients and grade 2 in one patient. Additionally, heterotopic ossifications rated as grade I were found in two patients.

\section{Discussion}

At present, there is considerable conflicting evidence in the literature regarding the treatment of comminuted and displaced fractures of the radial head $[2,3,6,19]$. Although RHR as early treatment has been reported with satisfactory to poor outcomes, the radiocapitellar contact is seriously damaged, leading to the instability of valgus or posterolateral rotation, decreased strength, and degenerative changes in elbow and wrist [20]. Consequently, RHA and ORIF are preferable in restoring the radiocapitellar relationship. Several excellent short to midterm results have been reported in comminuted radial head fractures treated with RHA, which provides an alternative surgical approach but have inherent drawbacks, such as heterotopic ossification, joint stiffness, hardware loosening, and radiocapitellar osteoarthritis, particularly when the prosthesis overstuffs the radiocapitellar joint and further alters the biomechanics of the radial head [21-23].

Biomechanical researches have proved the critical role of the radial head as a secondary stabilizer against both valgus and posterolateral instability of the elbow, especially in those cases with the medial collateral ligament or interosseous membrane of the forearm injury [4,24]. Thus, being aware of the importance of the radial head for elbow function, ORIF should be the initial surgical solution to retain the integrity of the fractured radial head, so as to maintain normal articulation and bone stock. If ORIF fails early, it can still be converted into RHA. Compared with RHR in the present study, seven patients except one with PLRI in the present study obtained good ROM of elbow and functional outcomes by ORIF. The results were accordant with previous views that ORIF was an excellent option for Mason type III fractures with a low level of associated injury [25]. However, following initial reconstruction of fractured radial head and avulsed ligament, the ROM of elbow and functional score of one patient with PLRI were inferior to the remaining cases, which mainly attributed to the nature of these complicated injuries.

A majority of previous studies have reported that comminuted radial head fractures with operative fixation have a high failure rate, nonunion, and osteonecrosis [26]. Moreover, severe Mason type-III radial head fractures are usually defined as "unsalvageable" or "unrepairable" type, which appears impossible to be treated with ORIF [27]. Nevertheless, the progress in implant technology and new fixation techniques have gradually overturned the established views. Particularly, on-table reconstruction technique has been 
reported and verified clinically. Several studies that exclusively focus on such technique for the complex radial head fractures have revealed satisfactory functional outcomes [7-9]. This technique is not limited to the narrowing anatomical joint space, and allows the reconstructed radial head to replant with the use of plate, which should be worthy of supporting and popularizing. However, in view of the different characteristics of comminuted radial head fractures, difficult extent to fix fragments and a variety of internal implants, the stability of fixation in the reconstructed radial head tends to be discrepant and remains to be assessed in different cases.

Koslowsky et al [28] have compared four different fixation techniques for the reconstruction of Mason type III fractures in the sawbones model, and find that displacement of the radial head from the neck often occur during plate fixation. Similarly, this issue was observed in the present study after on-table reconstruction of the radial head, leading to a poor radiocapitellar contact and incongruity between the radial head and neck. It is well known that treatment of radial head fractures mainly aims to maximize elbow movement and stability to maintain function [29]. Correct alignment of the radial neck to the reconstructed radial head is crucial for normal rotation in the proximal radioulnar joint. Therefore, only reconstruction of the radial head fails to meet the treatment criterion. Furthermore, many biomechanical studies have shown that the stability in the coronal and sagittal plane largely depends on the anatomical restoration of the radiocapitellar relationship [30].

We analyzed that the unstable displacement might be correlated to the insufficient axial support provided by plate fixation. Both unfirm anchoring force and weak supporting strength from proximal screws to the reconstructed radial head were critical factors. Primarily, the reconstructed radial head with Kirschner wires might block inserting of the proximal screws, and the plate must be placed at "safe-zone," which mainly limited the amounts, location, and direction of the screws. Moreover, the shape of fracture fragments was not uniform in different cases, so that the expected effects of manipulating on-table reconstruction remained uncertainties. The power drill, Kirschner wires, and proximal screws all probably would break the fragments and made reduction and fixation more complicated. Additionally, differences in plate type and operator's surgical experience could affect the definitive outcomes, which might be inconsistent with previously reported results. Conclusively, all above-mentioned factors might influence the anchoring force and supporting strength of screws, causing instability between the radial head and neck during plate fixation.

If plate fixation fails to offer reliable axial support, a hybrid technique combining with intramedullary pinning seems useful to compensate for that deficiency. The intramedullary pinning technique is always manipulated in children radial neck fractures, which allows minimally invasive and is easily performed, representing a favorable result with few complications [31]. Sandmann et al [32] first report that intramedullary pinning not only suits to radial neck fractures in children but satisfactory in adults. A series of superior results have confirmed the feasibility of adult radial neck fractures fixed with intramedullary pinning. Gao et al [33] has conceived a hypothesis that radial neck component could be stabilized by intramedullary pinning fixation following open reduction and plates or screws fixation in radial head fractures. This hybrid technique was manipulated and verified in our study, by which an 
additional axial support was obtained in the medullary cavity to prevent the displacement of reconstructed radial head from neck, and maintain restoration of radiocapitellar contact. Also, the use of elastic nail was modified in the present study. The proximal part of the elastic nail was bent, and formed three-point supporting consisting of the nail tip, bending point and nail bottom, which further enhance the axial support to avoid longitudinal displacement of the radial head.

Early failure in plate fixation is not infrequent, particularly in severe comminuted fractures. Ring et al [26] have shown that 3 of 14 fractures $(21.4 \%)$ with more than three articular fragments have failure in plate fixation within the first month. Therefore, if there is lack of stability and loss of normal alignment between the reconstructed radial head and neck during plate fixation, incorporating the use of intramedullary pining appears applicable. Moreover, stable fixation in fracture site allows patient to functional exercise early and promotes bone union. Kiran Kumar et al [8] have reported that only 3 of the 6 patients treated with on-table reconstruction achieve fracture healing after a mean follow-up of 25 months, and one case occurs avascular necrosis of radial head. There is a strong possibility that these complications are attributed to the unstable plate fixation. Comparatively, in the present study, seven patients following additional intramedullary pinning fixation had obtained bone union without avascular necrosis of radial head observed during follow-up. This hybrid technique preserved the integrity of radial head and provided satisfactory elbow function compared with RHR, which was available for selection following unstable plate fixation.

Objectively speaking, there are still some risks and limitations to this technique. Firstly, after plate fixation, distal screws may block insertion of elastic nail. Actually, the axial direction of nail tip is designed to have an angle of approximately 30 degrees with nail shaft. During elastic nail insertion, we suggest that the direction in which nail tip point on the coronal plane should try to keep parallel with the distal screws, and in this way that nail tip can pass through distal screws with the minimum contact area. Additionally, the width of the proximal radial medullary cavity is about $6-7 \mathrm{~mm}$, and the diameter of distal screws we used is $1.5 \mathrm{~mm}$, thus there is a sufficient interspace between screws and medial cortical bone to allow 2.0 $\mathrm{mm}$-sized elastic nail insertion. Secondly, inserting the elastic nail may penetrate the articular surface through the inter-fragmental gap, and cause failure in intramedullary fixation. In addition, the nail tip might potentially lift the fracture fragments, with the articular surface secondary displaced. Therefore, this technique needs the accumulation of operating experiences and learning curves. We suggest using the intraoperative fluoroscopy to control the inserting depth of nail, till the nail tip has advanced to the subchondral bone. Due to a small number of cases, the value of our study is limited. Moreover, the axial support provided by the elastic nail requires more quantitative analysis, patient accumulation, and further biomechanical exploration.

In conclusion, on-table technique offers an optional solution in the surgical treatment of comminuted radial head fractures. However, if there is unstable displacement from the reconstructed radial head to the neck during plate fixation, intramedullary pinning technique can be selected and applied as a remedial option. Indeed, this hybrid approach availably decrease the risks of failure in fixation and provide a good clinical result. 


\section{Abbreviations}

RHR

Radial head resection

RHA

Radial head arthroplasty

ORIF

Open reduction and internal fixation

TEN

Titanium elastic nails

ROM

Range of motion

CT

Computed tomography

VAS

Visual Analog Scale score

ESAS

Elbow Self-Assessment score

MEPS

Mayo Elbow Performance score

DASH

Disabilities of the Arm, Shoulder, and Hand

PLRI

Posterolateral rotational instability

\section{Declarations}

Acknowledgements: Not applicable

Ethics approval and consent to participate: This study was conducted in accordance with the principles of the Declaration of Helsinki, and approved by the medical ethics committee of No. 971 Hospital of the People's Liberation Army (PLA).

Consent for publication: Not applicable

Competing interests: The authors declare that they have no competing interests

Funding: No funding was received

Availability of data and material: Not applicable 
Authors' Information: Xu Gao ${ }^{1}$, Shi-you Dai ${ }^{2}$, Hai-lei Yin ${ }^{3 *}, \mathrm{Fei} \mathrm{Li}^{3}$, Yong-qiang Sui ${ }^{4}$, Rui Huang ${ }^{3}$, Hai-yu Fan $^{5}$

${ }^{1}$ Department of Orthopaedic Surgery, Qingdao University, Qingdao City, 266071, P.R. China

${ }^{2}$ Department of Bone, Joint and Sports Medicine, East District, Qingdao Municipal Hospital, Qingdao City, 266071, P.R. China

${ }^{3}$ Department of Second Orthopaedic Surgery, No. 971 Hospital of the People's Liberation Army (PLA), Qingdao City, 266071, P.R. China

${ }^{4}$ Department of State Key Laboratory for Marine Corrosion and Protection, Luoyang Ship Material Research Institute, Qingdao City, 266071, P.R. China

${ }^{5}$ Department of Burn and Plastic Surgery, No. 971 Hospital of the People's Liberation Army (PLA), Qingdao City, 266071, P.R. China

*Corresponding author: Hai-lei Yin, Doctor of Medicine, Department of Second Orthopaedic Surgery, No. 971 Hospital of the People's Liberation Army (PLA), No.22, Min-Jiang Rd, Qingdao 266071, P.R. China. Email: stonethrough@yeah.net

Authors' contributions: Hai-lei Yin, the corresponding author, offered idea about this study and provided research data. Xu Gao analyzed research data, and was a major contributor in writing the manuscript; The remaining authors including Shi-you Dai, Fei Li, Yong-qiang Sui, Rui Huang, Hai-yu Fan, participated in the correction and proofreading of this article. All authors had read and approved the final manuscript, and ensure that this is the case.

\section{References}

1. Mason ML. Some observations on fractures of the head of the radius with a review of one hundred cases. Br J Surg. 1954; 42:123-32. DOI:10.1002/bjs.18004217203.

2. Kodde IF, Kaas L, Flipsen M, Bekerom MP, Eygendaal D. Current concepts in the management of radial head fractures. World J Orthop. 2015;6: 954-60. DOI: 10.5312/wjo.v6.i11.954.

3. Miller G, Humadi A, Unni R, Hau R. Surgical management of Mason type III radial head fractures. Indian J Orthop. 2013;47: 323-32. DOI: 10.4103/0019-5413.114907.

4. Morrey BF, Tanaka S, An KN. Valgus stability of the elbow: a definition of primary and secondary constraints. Clin Orthop. 1991;265: 187-95.

5. Burkhart KJ, Mueller LP, Krezdorn D, Appelmann P, Prommersberger KJ, Sternstein W, Rommens PM. Stability of radial head and neck fractures: a biomechanical study of six fixation constructs with 
consideration of three locking plates. J Hand Surg Am. 2007; 32:1569-75. DOI:

10.1016/j.jhsa.2007.08.023.

6. Gokaraju K, Domos P, Aweid O, Fisher R, White A, Rensburg LV, Kang SN. Mid-term outcomes of surgical management of complex, isolated radial head fractures: a multicentre collaboration. Eur $\mathrm{J}$ Orthop Surg Traumatol. 2020. DOI: 10.1007/s00590-020-02709-z.

7. Businger A, Ruedi TP, Sommer C. On-table reconstruction of comminuted fractures of the radial head. Injury. 2010;41: 583-8. DOI: 10.1016/j.injury.2009.10.026.

8. Kiran Kumar GN, Sharma G, Farooque K, Sharma V, Jain V, Singh R, Morey V. On-table reconstruction and fixation of Mason type III radial head fractures. Chin J Traumatol. 2015;18: 288-92. DOI: 10.1016/j.cjtee.2015.11.005.

9. Everding J, Raschke M J, Polgart P, Grüneweller N, Wähnert D, Schliemann B. Ex situ reconstruction of comminuted radial head fractures: is it truly worth a try?. Arch Orthop Trauma Surg. 2019;139: 172329. DOI: $10.1007 / \mathrm{s} 00402-019-03250-3$.

10. Chen DW, Hu WK, Zhou JQ. Use of surgical glue for Mason type III radial head fractures: A case report. Medicine (Baltimore). 2019; 98: e15863. DOI:10.1097/MD.0000000000015863.

11. Guo L, Li R, Yang X, Yu C, Gui F. Polylactide pins can effectively fix severely comminuted and unsalvageable radial head fracture: A retrospective study of 40 patients. Injury 2020; 51:2253-8. DOI: 10.1016/j.injury.2020.07.041.

12. Fedorka CJ, Oh LS. Posterolateral rotatory instability of the elbow. Curr Rev Musculoskelet Med. 2016; 9:240-6. DOI: 10.1007/s12178-016-9345-8.

13. Josten C, Freitag S. Monteggia and Monteggia-like-lesions: Classification, Indication, and Techniques in Operative Treatment. Eur J Trauma Emerg Surg. 2009; 35: 296-304. DOI: 10.1007/s00068-0088028-6.

14. Beirer M, Friese H, Lenich A, Crönlein M, Sandmann GH, Biberthaler P, Kirchhoff C, Siebenlist S. The Elbow Self-Assessment Score (ESAS): development and validation of a new patient-reported outcome measurement tool for elbow disorders. Knee Surg Sports Traumatol Arthrosc. 2017; 25:2230-6. DOI: 10.1007/s00167-015-3647-z.

15. Morrey BF, Bryan RS, Dobyns JH, Linscheid RL. Total elbow arthroplasty. A five-year experience at the Mayo Clinic. J Bone Joint Surg Am. 1981; 63:1050-63.

16. Gummesson C, Ward MM, Atroshi I. The shortened disabilities of the arm,shoulder and hand questionnaire (Quick DASH): validity and reliability based on responses within the full-length DASH. BMC Musculoskelet Disord. 2006; 7: 44. DOI: 10.1186/1471-2474-7-44. 
17. Hastings $\mathrm{H}$, Graham TJ. The classification and treatment of heterotopic ossification about the elbow and forearm. Hand Clin. 1994;10: 417-37.

18. Broberg MA, Morrey BF. Results of treatment of fracture-dislocations of the elbow. Clin Orthop Relat Res. 1987;216: 109-19.

19. Jordan RW, Jones AD. Radial Head Fractures. Open Orthop J. 2017;11: 1405-16. DOI: $10.2174 / 1874325001711011405$.

20. Sanchez-Sotelo J, Romanillos O, Garay EG. Results of acute excision of the radial head in elbow radial head fracture-dislocations. J Orthop Trauma. 2000; 14:354-8. DOI: 10.1097/00005131200006000-00009.

21. Al-Burdeni $S$, Abuodeh $Y$, Ibrahim $T$, Ahmed $G$. Open reduction and internal fixation versus radial head arthroplasty in the treatment of adult closed comminuted radial head fractures (modified Mason type III and IV). Int Orthop. 2015; 39:1659-64. DOI: 10.1007/s00264-015-2755-1.

22. Arbter $D$, Piatek S, Probst A, Holmenschlager F, Winckler $S$. Results after Judet radial head prosthesis for non-reconstructable radial head fractures. Der Unfallchirurg. 2012; 115:1000-8. DOI:

\subsection{7/s00113-011-1990-1.}

23. Delclaux S, Lebon J, Faraud A, Toulemonde J, Bonnevialle N, Coulet B, Mansat P. Complications of radial head pros-theses. Int Orthop. 2015; 39: 907-13. DOI: 10.1007/s00264-015-2689-7.

24. Schneeberger AG, Sadowski MM, Jacob HA. Coronoid process and radial head as posterolateral rotatory stabilizers of the elbow. J Bone J Surg. 2004; 86:975-82. DOI:10.2106/00004623200405000-00013.

25. Lee YS, Kang YH, Chung YG, Shin SH. Open reduction and internal fixation for Mason type III radial head fractures: Is it different from that for Mason type II fractures?. J Orthop Surg (Hong Kong). 2018; 26. DOI: $10.1177 / 2309499018802506$.

26. Ring D. Open reduction and internal fixation of fractures of the radial head. Hand Clin. 2004; 20:41527. DOl: $10.1016 /$ j.hcl.2004.06.001.

27. Catellani F, De Caro F, De Biase CF, Perrino VR, Usai L, Triolo V, Ziveri G, Fiorentino G. Radial Head Resection versus Arthroplasty in Unrepairable Comminuted Fractures Mason Type III and Type IV: A Systematic Review. Biomed Res Int. 2018; 2018:4020625. DOI:10.1155/2018/4020625.

28. Koslowsky TC, Mader K, Dargel J, Koebke J, Hellmich M, Pennig D. Reconstruction of a Mason type-III fracture of the radial head using four different fixation techniques. An experimental study. J Bone Joint Surg Br. 2007; 89:1545-50. DOI: 10.1302/0301-620X.89B11.19433.

29. Charalambous CP, Stanley JK, Mills SP, Hayton1MJ, Hearnden1 A, Trail I, Gagey O. Comminuted radial head fractures: aspects of current management. J Shoulder Elbow Surg. 2011; 20:996- 
1007. DOI: 10.1016/j.jse.2011.02.013.

30. Ruchelsman DE, Christoforou D, Jupiter JB. Current Concepts Review: Fracture of the radial head and neck. J Bone Joint Surg Am. 2013; 95: 469-78. DOI: 10.2106/JBJS.J.01989.

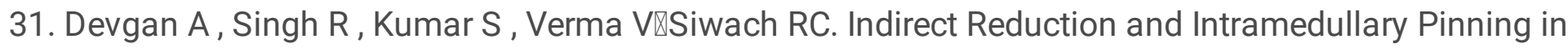
Severely Displaced Radial Neck Fractures in Children. J Biomech. 2011; 45: S537. DOI:

\subsection{6/S0021-9290(12)70538-9.}

32. Yallapragada RK, Maripuri SN. Radial neck fractures in children: A surgical tip using the Metaizeau technique to improve stability of the reduction. J Orthop. 2019; 17: 127-33. DOI:

\subsection{6/j.jor.2019.06.001.}

33. Gao X, Yin HL, Zhou GJ. Minimally Invasive Treatment of Mason Type II Radial Head Fracture by Intramedullary Pinning. Orthop Surg. 2019; 11:879-85. DOI: 10.1111/os.12540.

\section{Tables}

Table 1. Details of patient demographics

\begin{tabular}{lll}
\hline & ORIF (experimental) & RHR (control) \\
\hline Patients [15] & 7 & 8 \\
Male [9] & 4 & 6 \\
Female [6] & 3 & 2 \\
Mean \pm SD age [years] & $35.2 \pm 3.6$ & $41.6 \pm 2.1$ \\
Side of the injury [15] & - & - \\
$\quad$ Right [11] & 5 & 6 \\
$\quad$ Left [4] & 2 & 2 \\
Mean \pm SD time of follow-up [months] & $37.2 \pm 5.8$ & $33.2 \pm 7.1$ \\
Mode of injury [15] & - & - \\
$\quad$ Falling injury [5] & 3 & 2 \\
$\quad$ Traffic accident [10] & 4 & 6 \\
\hline
\end{tabular}

Table 2. Intraoperative comparison of the groups

\begin{tabular}{llll}
\hline & ORIF (experimental) & RHR (control) & $p$ \\
\hline Mean \pm SD operating time [min] & $128.7 \pm 10.3$ & $122.5 \pm 15.7$ & 0.523 \\
Mean \pm SD blood loss [ml] & $135.3 \pm 13.2$ & $129.1 \pm 16.5$ & 0.893 \\
Mean \pm SD fluoroscopy times [time] & $11.5 \pm 4.2$ & $3.7 \pm 1.6$ & 0.000 \\
\hline
\end{tabular}

Table 3. The clinical, functional and radiographic outcomes of the groups 


\begin{tabular}{llll}
\hline & ORIF (experimental) & RHR (control) & $p$ \\
\hline Mean \pm SD VAS & $2.5 \pm 0.8$ & $3.1 \pm 1.4$ & 0.872 \\
Mean \pm SD MEPS & $85.6 \pm 7.3$ & $78.2 \pm 2.9$ & 0.002 \\
Mean \pm SD DASH & $9.7 \pm 1.1$ & $14.6 \pm 2.8$ & 0.009 \\
Mean \pm SD arc of elbow motion & $131.4 \pm 7.2$ & $121.6 \pm 3.8$ & 0.008 \\
Mean \pm SD extension deficit & $13.2 \pm 2.6$ & $20.8 \pm 1.9$ & 0.001 \\
Mean \pm SD pronation & $73.8 \pm 7.1$ & $74.4 \pm 7.6$ & 0.457 \\
Mean \pm SD supination & $67.7 \pm 4.6$ & $70.0 \pm 3.8$ & 0.229 \\
Arthritis [6] & - & - & - \\
$\quad$ Grade 1 [5] & 2 & 3 & - \\
$\quad$ Grade 2 [1] & 0 & 1 & - \\
Heterotopic ossifications rated as Grade 1[4] & 2 & 2 & - \\
Elbow Self-Assessment [15] & - & - & - \\
$\quad$ Very good [1] & 1 & 0 & - \\
Good [5] & 2 & 3 & - \\
Satisfied [5] & 3 & 2 & \\
Sufficient [4] & 1 & 3 & \\
& & & \\
\end{tabular}

VAS, Visual Analogue Scale; MEPS, Mayo Elbow Performance Score; DASH, Disabilities of the arm, shoulder and hand

Figures 


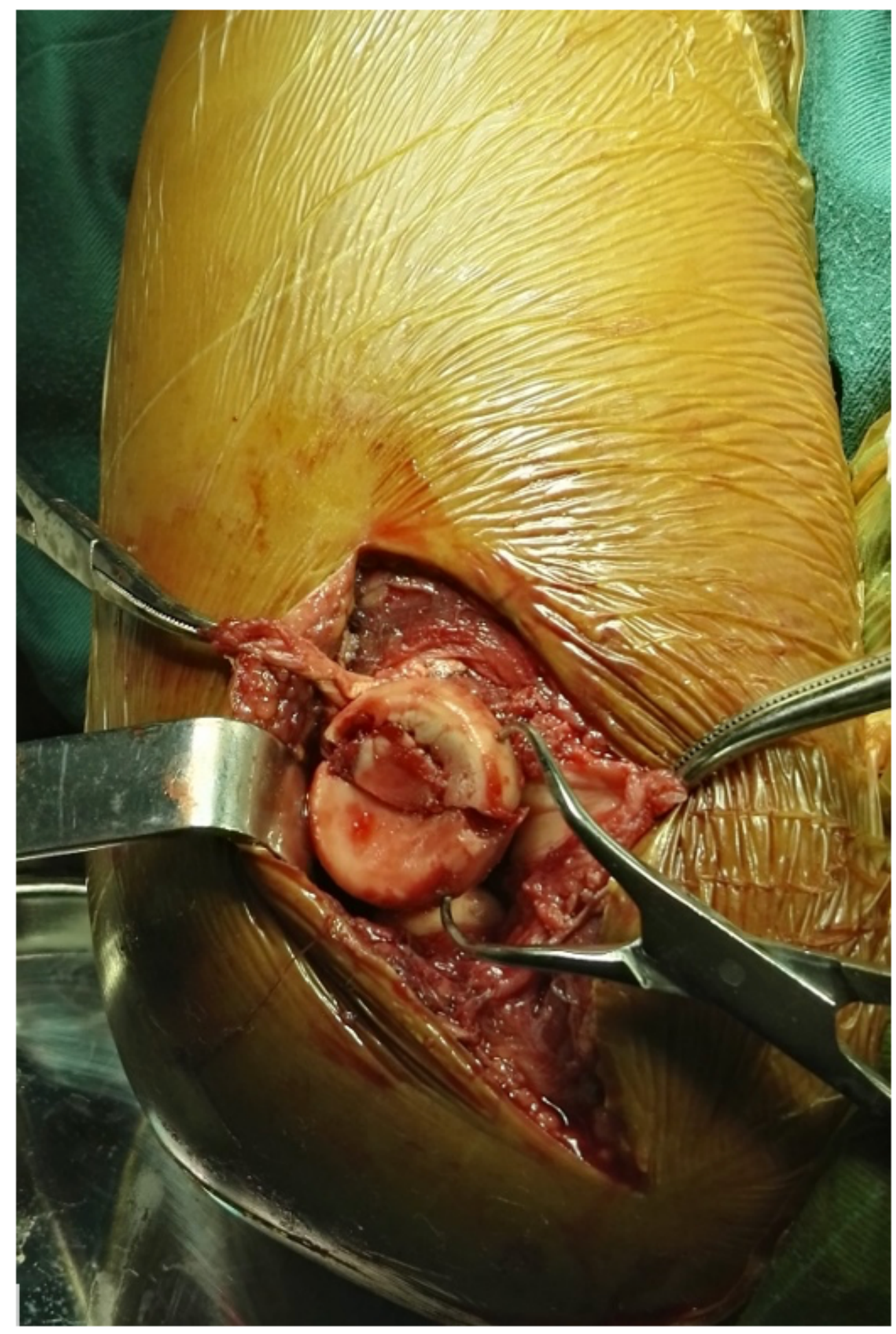

Figure 1

The reconstructed radial head was replaced to the elbow joint. 


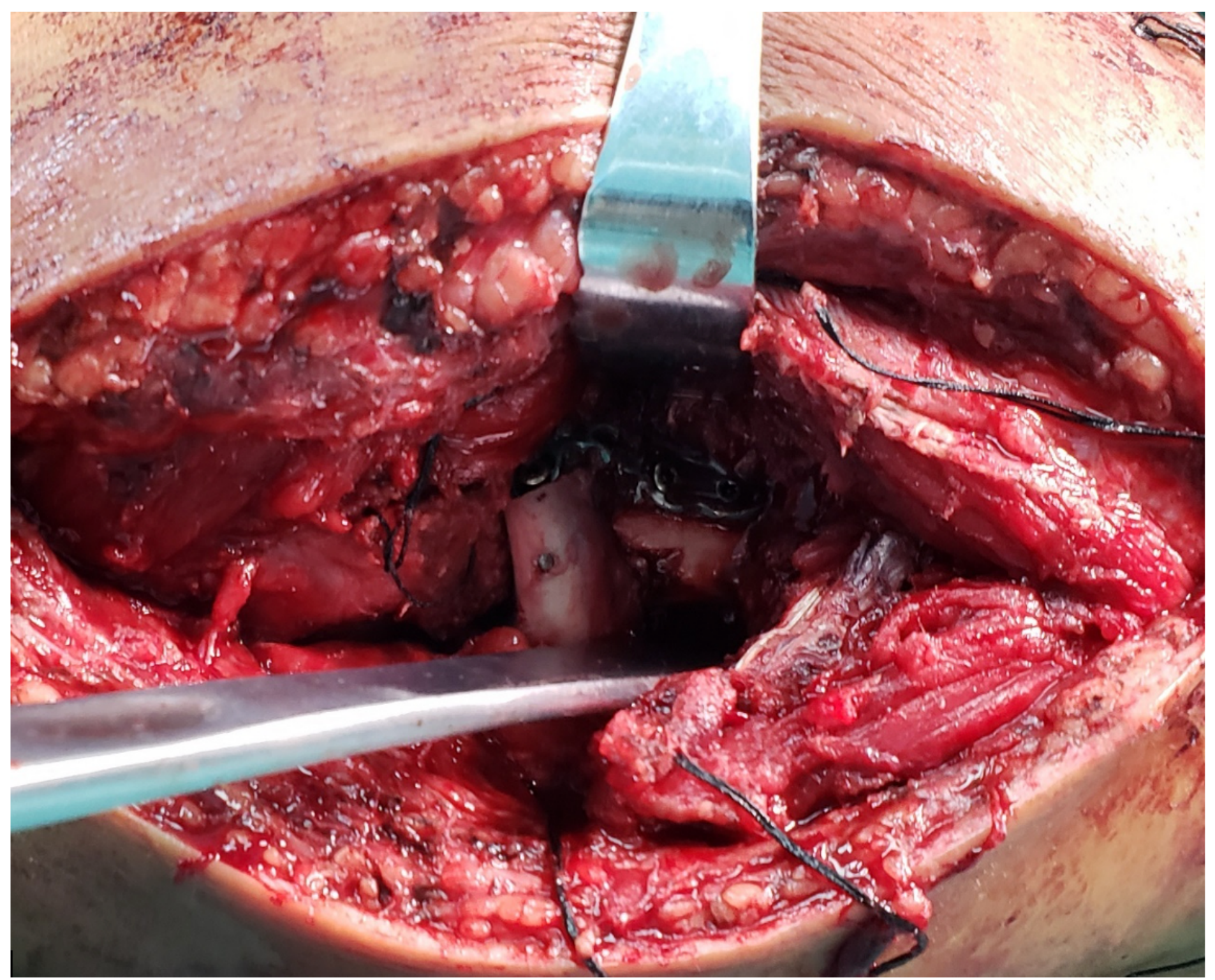

\section{Figure 2}

Displacement of the radial head from the neck with a poor alignment. 


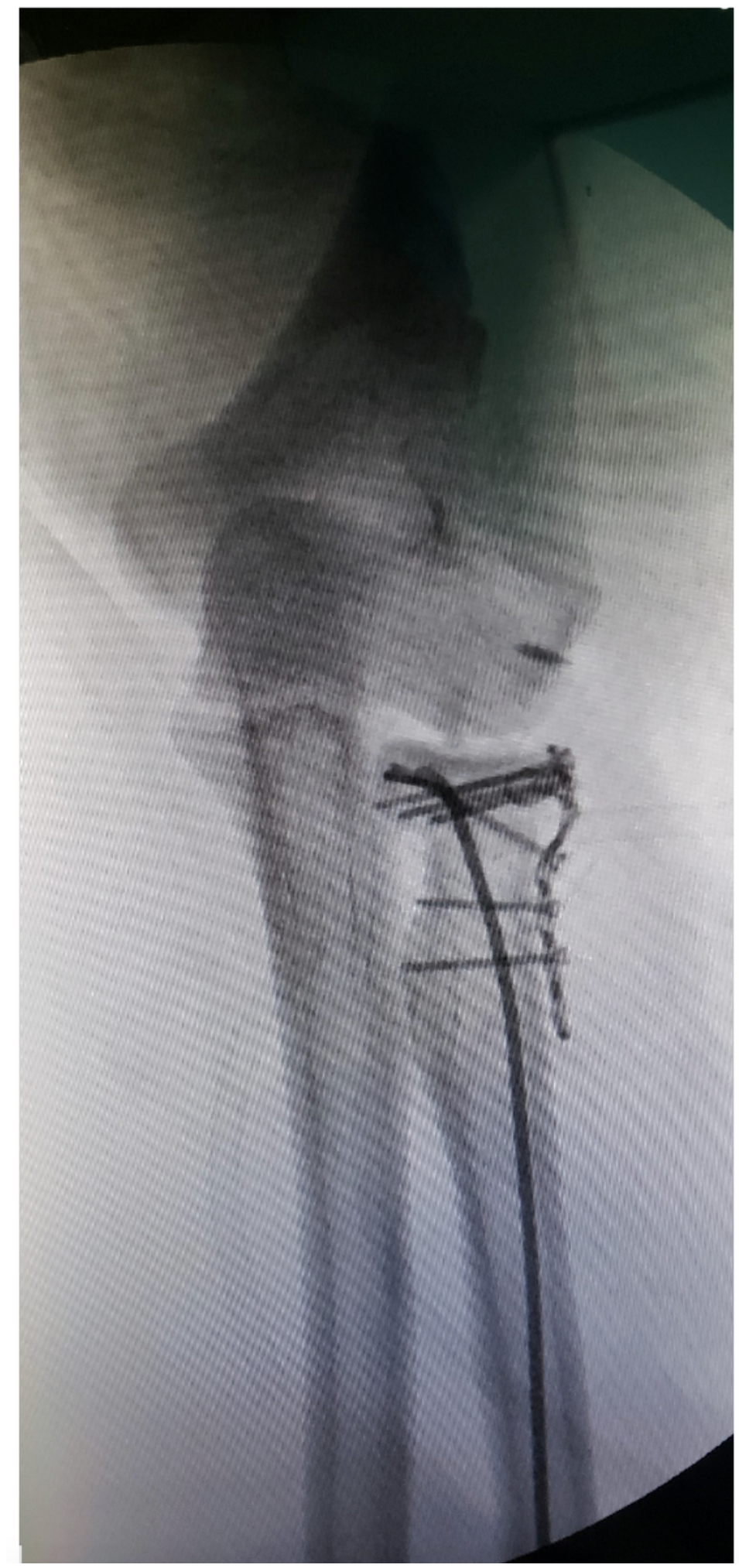

\section{Figure 3}

A satisfactory restoration of alignment between the radial head and neck after intramedullary pinning fixation. 


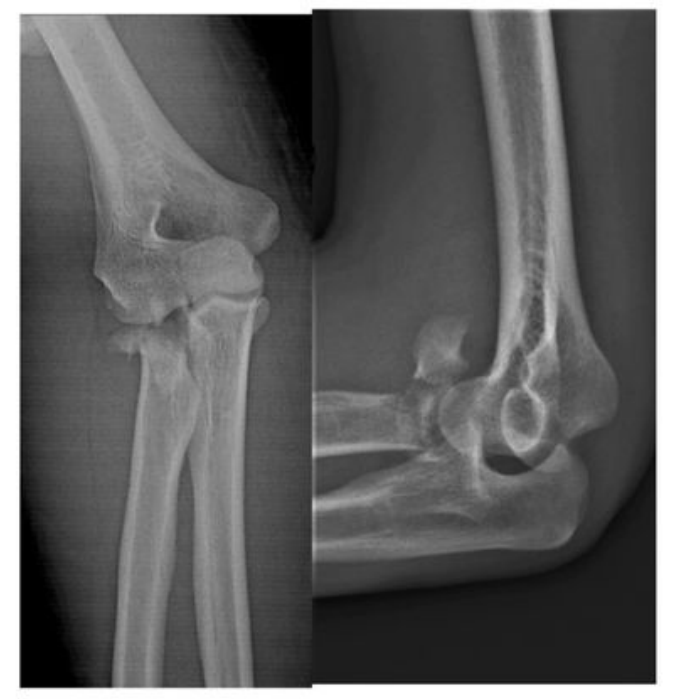

a

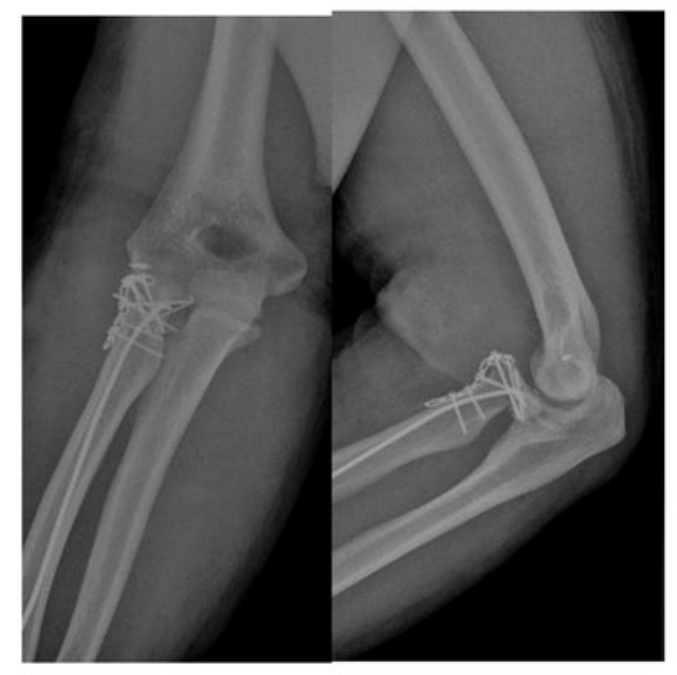

b

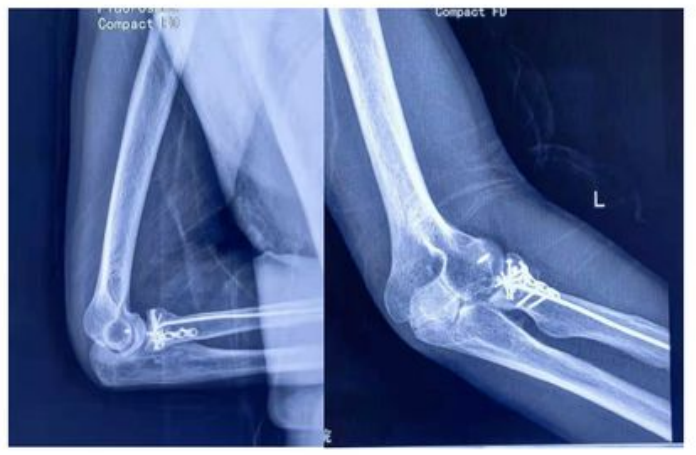

c

Figure 4

A left radial head fracture in a 47-year-old man. a. Preoperative radiograph showed Mason type III radial head fracture with PLRI injury. b. Following extramedullary plate and intramedullary pinning fixation, postoperative radiograph revealed a good restoration of alignment between the radial head and neck. c. Anteroposterior and lateral radiograph showed bone union at 12 months after surgery 


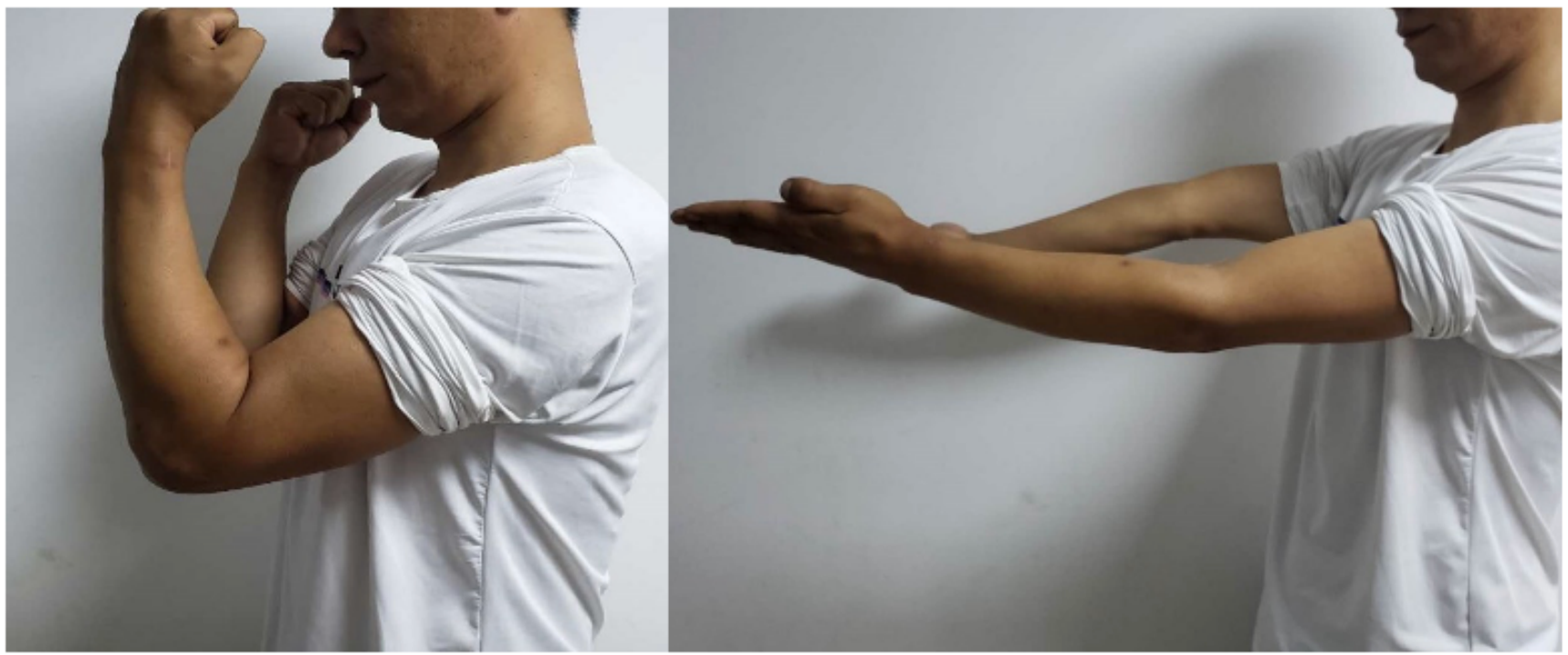

$\mathbf{a}$

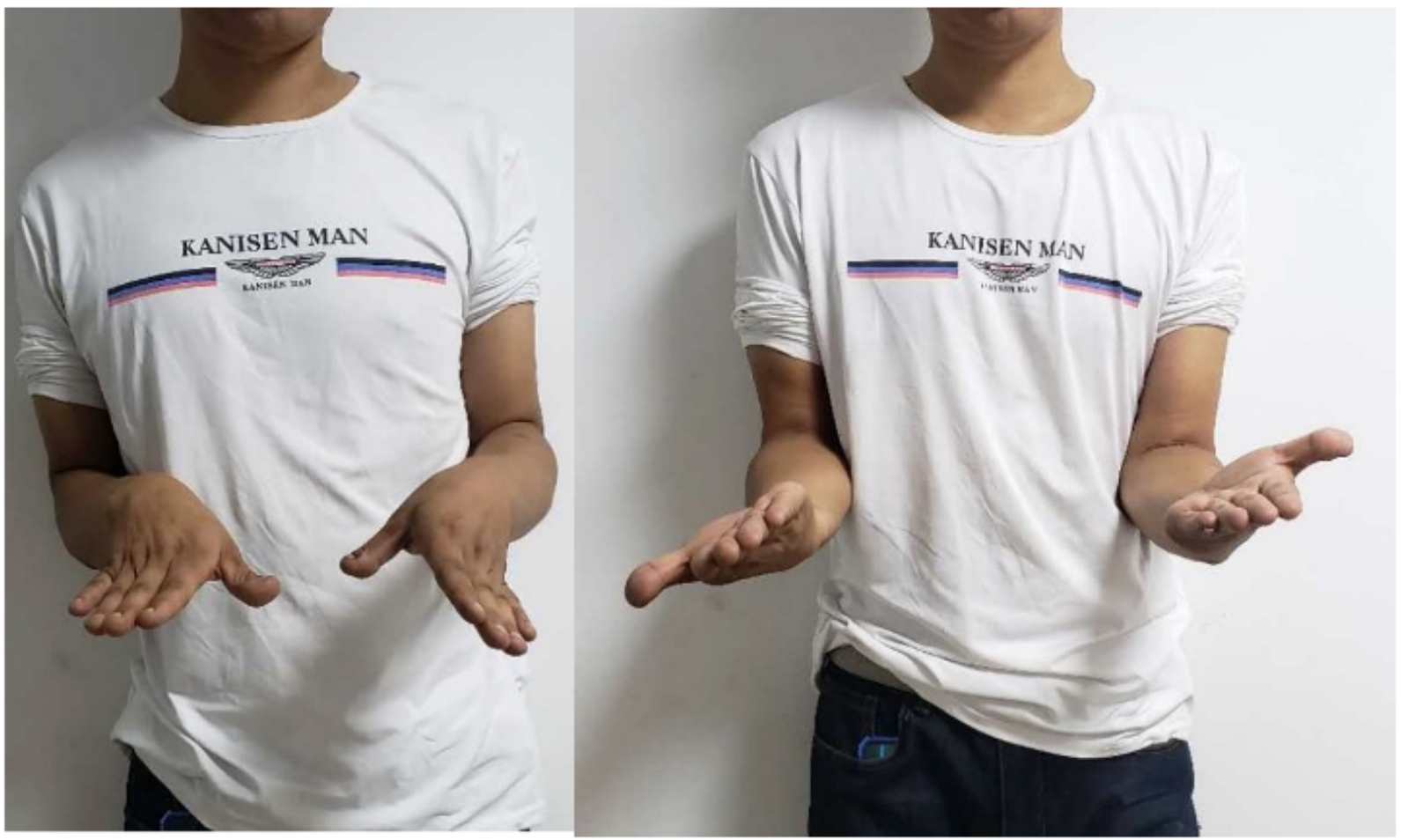

b

Figure 5

Functional outcomes at the final follow-up a. Compared with the unaffected sides, the ROM of elbow was insufficient, with the arc of elbow movement of $95^{\circ} \mathrm{b}$. The forearm pronation was acceptable but the supination of $65^{\circ}$ was inadequate. 